\title{
Approximate symmetries in nonlinear viscoelastic media
}

\author{
Marianna Ruggieri ${ }^{* *}$ and Antonino Valenti
}

\author{
"Correspondence: \\ marianna.ruggieri@unikore.it \\ ${ }^{1}$ Facoltà di Ingegneria e \\ Architettura, Università di Enna \\ 'Kore', Cittadella Universitaria, Enna, \\ 94100 , Italy \\ Full list of author information is \\ available at the end of the article
}

\begin{abstract}
Approximate symmetries of a mathematical model describing one-dimensional motion in a medium with a small nonlinear viscosity are studied. In a physical application, the approximate solution is calculated making use of the approximate generator of the first-order approximate symmetry.
\end{abstract}

MSC: Primary 35J25; 32A37; 43A15; secondary 35A58; 42B20

Keywords: existence; uniqueness and stability of solutions; partial differential equations; approximate method

\section{Introduction}

We consider the third-order partial differential equation

$$
w_{t t}=\left[\sigma\left(w_{x}\right)+\lambda\left(w_{x}\right) w_{t x}\right]_{x}
$$

where $\sigma$ and $\lambda$ are smooth functions, $w(t, x)$ is the dependent variable and subscripts denote partial derivative with respect to the independent variables $t$ and $x$.

Equation (1) can describe the behavior of a one-dimensional viscoelastic medium in which nonlinearities appear not only in the elastic part of the stress, but also in the viscoelastic one.

Some mathematical questions as the global existence, uniqueness and stability of solutions can be found in [1, 2]. Moreover, shear wave solutions are found in [3], where some explicit examples of blow-up for boundary value problems with smooth initial data are shown. A symmetry analysis and some exact solutions are shown in [4-6], while when $\lambda\left(w_{x}\right)=\lambda_{0}$, with $\lambda_{0}$ a positive constant, a symmetry analysis can be performed in [7-9].

It is well known that a small dissipation is able to prevent the breaking of the wave profile allowing to study the so called 'far field', and a technique widely used is the perturbation analysis performed by expanding the dependent variables in power series of a small parameter (may be a physical parameter or often artificially introduced). Having in mind to perform an 'approximate symmetry analysis', we introduce in (1) a small parameter $\varepsilon$, namely

$$
w_{t t}=f\left(w_{x}\right) w_{x x}+\varepsilon\left[\lambda\left(w_{x}\right) w_{t x}\right]_{x},
$$


with $f=\sigma^{\prime}$ (hereafter, a prime denotes derivative of a function with respect to the only variable upon which it depends). For $\varepsilon=0$, we recover the nonlinear wave equation

$$
w_{t t}=f\left(w_{x}\right) w_{x x}
$$

The combination of the Lie group theory and the perturbation analysis gives rise to the so-called approximate symmetry theories. The first paper on this subject is due to Baikov, Gazizov and Ibragimov [10]. Successively another method for finding approximate symmetries was proposed by Fushchich and Shtelen [11]. In the method proposed by Baikov, Gazizov and Ibragimov, the Lie operator is expanded in a perturbation series so that an approximate operator can be found. But the approximate operator does not reflect well an approximation in the perturbation sense; in fact, even if one uses a first-order approximate operator, the corresponding approximate solution could contain higher-order terms.

In the method proposed by Fushchich and Shtelen the dependent variables are expanded in a perturbation series; equations are separated at each order of approximation and the approximate symmetries of the original equations are defined to be the exact symmetries of the system coming out from equating to zero the coefficients of the smallness parameter. This method is consistent with the perturbation theory and yields correct terms for the approximate solutions but a 'drawback' is present: it is impossible to work in hierarchy, i.e., in the search for symmetries, there is a coupled system between the equations at several orders of approximation, therefore the algebra can increase enormously.

In this paper we work in the framework of the approximate method proposed in [12, 13], in which the expansions of the dependent variable are introduced also in the Lie group transformations so that one obtains an approximate Lie operator which permits to solve in hierarchy the invariance conditions starting from the classification of unperturbed equation (3). We obtain the symmetry classification of the functions $f\left(w_{x}\right)$ and $\lambda\left(w_{x}\right)$ through which equation (2) is approximately invariant and search for approximate solutions.

The plan of the paper is the following. The approximate symmetry method is introduced in the next section; the group classification via approximate symmetries is performed in Section 3; in Section 4, in a physical application, the approximate solution is calculated by means of the approximate generator of the first-order approximate group of transformations.

\section{Approximate symmetry method}

In general, any solution of (2) will be of the form $w=w(t, x, \varepsilon)$ and the one-parameter Lie group of infinitesimal transformations in the $(t, x, w)$-space of equation (2) can be considered in the following form:

$$
\begin{aligned}
& \hat{t}=t+a \xi^{1}(t, x, w(t, x, \varepsilon), \varepsilon)+\mathcal{O}\left(a^{2}\right), \\
& \hat{x}=x+a \xi^{2}(t, x, w(t, x, \varepsilon), \varepsilon)+\mathcal{O}\left(a^{2}\right), \\
& \hat{w}=w+a \eta(t, x, w(t, x, \varepsilon), \varepsilon)+\mathcal{O}\left(a^{2}\right),
\end{aligned}
$$

where $a$ is the group parameter. 
Let us suppose that $w(t, x, \varepsilon)$ and $\hat{w}(\hat{t}, \hat{x}, \varepsilon)$, analytic in $\varepsilon$, can be expanded in power series of $\varepsilon$, i.e.,

$$
\begin{aligned}
& w(t, x, \varepsilon)=w_{0}(t, x)+\varepsilon w_{1}(t, x)+\mathcal{O}\left(\varepsilon^{2}\right), \\
& \hat{w}(\hat{t}, \hat{x}, \varepsilon)=\hat{w}_{0}(\hat{t}, \hat{x})+\varepsilon \hat{w}_{1}(\hat{t}, \hat{x})+\mathcal{O}\left(\varepsilon^{2}\right),
\end{aligned}
$$

where $w_{0}$ and $w_{1}$ are some smooth functions of $t$ and $x ; \hat{w}_{0}$ and $\hat{w}_{1}$ are some smooth functions of $\hat{t}$ and $\hat{x}$.

Upon formal substitution of (7) in (2), equating to zero the coefficients of zero and first degree powers of $\varepsilon$, we arrive at the following system of PDEs:

$$
\begin{aligned}
L_{0}:= & w_{0 t t}-f\left(w_{0 x}\right) w_{0 x x}=0, \\
L_{1}:= & w_{1 t t}-f\left(w_{0 x}\right) w_{1 x x}-f^{\prime}\left(w_{0 x}\right) w_{0 x x} w_{1 x} \\
& -\lambda^{\prime}\left(w_{0 x}\right) w_{0 x x} w_{0 t x}-\lambda\left(w_{0 x}\right) w_{0 x x t}=0,
\end{aligned}
$$

where we have set

$$
\begin{array}{ll}
f\left(w_{0 x}\right)=\left.f\left(w_{x}\right)\right|_{\varepsilon=0}, & f^{\prime}\left(w_{0 x}\right)=\left.f^{\prime}\left(w_{x}\right)\right|_{\varepsilon=0}, \\
\lambda\left(w_{0 x}\right)=\left.\lambda\left(w_{x}\right)\right|_{\varepsilon=0}, & \lambda^{\prime}\left(w_{0 x}\right)=\left.\lambda^{\prime}\left(w_{x}\right)\right|_{\varepsilon=0} .
\end{array}
$$

Hence, $w_{0}$ is a solution of nonlinear wave equation (9) which we call unperturbed equation, while $w_{1}$ can be determined from the linear equation (10).

In order to have a one-parameter Lie group of infinitesimal transformations of the system (9)-(10), which is consistent with the expansions of the dependent variables (7) and (8), we introduce these expansions in the infinitesimal transformations (4)-(6). Upon formal substitution, equating to zero the coefficients of zero and first degree powers of $\varepsilon$, we get the following one-parameter Lie group of infinitesimal transformations in the $\left(t, x, w_{0}, w_{1}\right)$ space

$$
\begin{aligned}
& \hat{t}=t+a \xi_{0}^{1}\left(t, x, w_{0}\right)+\mathcal{O}\left(a^{2}\right), \\
& \hat{x}=x+a \xi_{0}^{2}\left(t, x, w_{0}\right)+\mathcal{O}\left(a^{2}\right), \\
& \hat{w}_{0}=w_{0}+a \eta_{0}\left(t, x, w_{0}\right)+\mathcal{O}\left(a^{2}\right), \\
& \hat{w}_{1}=w_{1}+a\left[\eta_{10}\left(t, x, w_{0}\right)+\eta_{11}\left(t, x, w_{0}\right) w_{1}\right]+\mathcal{O}\left(a^{2}\right),
\end{aligned}
$$

where we have set

$$
\begin{aligned}
& \xi_{0}^{i}\left(t, x, w_{0}\right)=\left.\xi^{i}(t, x, w(t, x, \varepsilon), \varepsilon)\right|_{\varepsilon=0}, \quad i=1,2, \\
& \eta_{0}\left(t, x, w_{0}\right)=\left.\eta(t, x, w(t, x, \varepsilon), \varepsilon)\right|_{\varepsilon=0}, \\
& \eta_{10}\left(t, x, w_{0}\right)+\eta_{11}\left(t, x, w_{0}\right) w_{1}=\left.\frac{d \eta}{d \varepsilon}\right|_{\varepsilon=0} .
\end{aligned}
$$

We give the following definition: We call approximate symmetries of equation (2) the (exact) symmetries of the system (9)-(10) through the one-parameter Lie group of infinitesimal transformations (11)-(14). Consequently, the one-parameter Lie group of infinitesimal 
transformations (11)-(14), the associated Lie algebra and the corresponding infinitesimal operator

$$
\begin{aligned}
X= & \xi^{1}\left(t, x, w_{0}\right) \frac{\partial}{\partial t}+\xi^{2}\left(t, x, w_{0}\right) \frac{\partial}{\partial x}+\eta\left(t, x, w_{0}\right) \frac{\partial}{\partial w_{0}} \\
& +\left[\eta_{10}\left(t, x, w_{0}\right)+\eta_{11}\left(t, x, w_{0}\right) w_{1}\right] \frac{\partial}{\partial w_{1}}
\end{aligned}
$$

are called the approximate Lie group, the approximate Lie algebra and the approximate Lie operator of equation (2), respectively.

Moreover, after putting

$$
X_{0}=\xi_{0}^{1}\left(t, x, w_{0}\right) \frac{\partial}{\partial t}+\xi_{0}^{2}\left(t, x, w_{0}\right) \frac{\partial}{\partial x}+\eta_{0}\left(t, x, w_{0}\right) \frac{\partial}{\partial w_{0}},
$$

the approximate Lie operator (18) can be rewritten as

$$
X=X_{0}+\left[\eta_{10}\left(t, x, w_{0}\right)+\eta_{11}\left(t, x, w_{0}\right) w_{1}\right] \frac{\partial}{\partial w_{1}}
$$

and $X_{0}$ can be regarded as the infinitesimal operator of unperturbed nonlinear wave equation (9) (or (3)).

It is worthwhile noticing that, thanks to the functional dependencies of the coordinates of the approximate Lie operator (18) (or (20)), now we are able to work in hierarchy in finding the invariance conditions of the system (9)-(10): firstly, by classifying unperturbed nonlinear wave equation (9) through the operator (19) and after by determining $\eta_{10}$ and $\eta_{11}$ from the invariance condition that follows by applying the operator (20) to the linear equation (10). In fact the invariance condition of the system (9)-(10) reads

$$
\begin{aligned}
& \left.X_{0}^{(2)}\left(L_{0}\right)\right|_{L_{0}=0}=0, \\
& \left.X^{(3)}\left(L_{1}\right)\right|_{L_{0}=0, L_{1}=0}=0,
\end{aligned}
$$

where $X_{0}^{(2)}$ and $X^{(3)}$ are the second and third extensions of the operators $X_{0}$ and $X$, respectively.

\section{Group classification via approximate symmetries}

The classification of equation (9) is well known (see for details Ibragimov [14] and bibliography therein). From (21), we arrive at the following result:

$$
\begin{aligned}
& \xi_{0}^{1}=a_{5} t^{2}+a_{3} t+a_{1}, \\
& \xi_{0}^{2}=a_{4} x+a_{2}, \\
& \eta_{0}=\left(a_{5} t+a_{6}\right) w_{0}+a_{7} t x+a_{8} t+a_{9} x+a_{10}, \\
& {\left[\left(a_{6}-a_{4}\right) w_{0 x}+a_{9}\right] f^{\prime}\left(w_{0 x}\right)-2\left(a_{4}-a_{3}\right) f\left(w_{0 x}\right)=0,} \\
& \left(a_{5} w_{0 x}+a_{7}\right) f^{\prime}\left(w_{0 x}\right)+4 a_{5} f\left(w_{0 x}\right)=0,
\end{aligned}
$$

where $a_{i}, i=1,2, \ldots, 10$ are constants. 
Taking (23)-(27) into account, from (22) we obtain the following additional conditions:

$$
\begin{aligned}
& a_{5}=a_{7}=0, \\
& \eta_{10}=a_{11} t+a_{12}, \\
& \eta_{11}=a_{13}, \\
& {\left[\left(a_{6}-a_{4}\right) w_{0 x}+a_{9}\right] \lambda^{\prime}\left(w_{0 x}\right)-\left(2 a_{4}-a_{3}-a_{6}+a_{13}\right) \lambda\left(w_{0 x}\right)=0,}
\end{aligned}
$$

with $a_{11}, a_{12}$ and $a_{13}$ being constants.

After observing that conditions (28) impose restrictions upon $X_{0}$, summarizing we have to manage the following relations:

$$
\begin{aligned}
& \xi_{0}^{1}=a_{3} t+a_{1}, \\
& \xi_{0}^{2}=a_{4} x+a_{2}, \\
& \eta_{0}=a_{6} w_{0}+a_{8} t+a_{9} x+a_{10}, \\
& \eta_{10}=a_{11} t+a_{12}, \\
& \eta_{11}=a_{13}, \\
& {\left[\left(a_{6}-a_{4}\right) w_{0 x}+a_{9}\right] f^{\prime}\left(w_{0 x}\right)-2\left(a_{4}-a_{3}\right) f\left(w_{0 x}\right)=0,} \\
& {\left[\left(a_{6}-a_{4}\right) w_{0 x}+a_{9}\right] \lambda^{\prime}\left(w_{0 x}\right)-\left(2 a_{4}-a_{3}-a_{6}+a_{13}\right) \lambda\left(w_{0 x}\right)=0 .}
\end{aligned}
$$

For $f$ and $\lambda$ arbitrary functions, we obtain

$$
a_{6}=a_{4}=a_{3}, \quad a_{9}=a_{13}=0
$$

and we call the associate seven-dimensional Lie algebra the approximate principal Lie algebra of equation (2). We denote it by $\mathcal{A p p r o x} \mathcal{L}_{\mathcal{P}}$ and it is spanned by the seven operators

$$
\begin{aligned}
& X_{1}=\frac{\partial}{\partial t}, \quad X_{2}=\frac{\partial}{\partial x}, \quad X_{3}=\frac{\partial}{\partial w_{0}}, \quad X_{4}=t \frac{\partial}{\partial w_{0}}, \\
& X_{5}=t \frac{\partial}{\partial t}+x \frac{\partial}{\partial x}+w_{0} \frac{\partial}{\partial w_{0}}, \quad X_{6}=\frac{\partial}{\partial w_{1}}, \quad X_{7}=t \frac{\partial}{\partial w_{1}} .
\end{aligned}
$$

The classification of $f\left(w_{0 x}\right)$ and $\lambda\left(w_{0 x}\right)$ with the corresponding extensions of $\mathcal{A p p r o x} \mathcal{L}_{\mathcal{P}}$ arising from (32)-(38) is reported in Table 1.

Table 1 Classification of $f\left(w_{0_{x}}\right)$ and $\lambda\left(w_{0 x}\right)$ with the corresponding extensions of $\mathcal{A p p r o x} \mathcal{L}_{\mathcal{P}}$

\begin{tabular}{lll}
\hline Case & Forms of $\boldsymbol{f}\left(\boldsymbol{w}_{\mathbf{0}_{\mathbf{x}}}\right)$ and $\boldsymbol{\lambda}\left(\boldsymbol{w}_{\mathbf{0}_{\mathbf{x}}}\right)$ & Extensions of $\boldsymbol{A p p r o x} \mathcal{L}_{\mathcal{P}}$ \\
\hline 1 & $f\left(w_{0 x}\right)=f_{0} e^{\frac{2}{p} w_{0 x}}$ & $x_{8}=x \frac{\partial}{\partial x}+\left(w_{0}+p x\right) \frac{\partial}{\partial w_{0}}+s w_{1} \frac{\partial}{\partial w_{1}}$ \\
$\lambda\left(w_{0 x}\right)=\lambda_{0} e^{\frac{1+s}{p} w_{0 x}}$ & \\
& $f\left(w_{0 x}\right)=f_{0}\left(w_{0 x}+q\right)^{\frac{2}{p}}$ & $x_{8}=x \frac{\partial}{\partial x}+\left[(1+p) w_{0}+p q x\right] \frac{\partial}{\partial w_{0}}+s w_{1} \frac{\partial}{\partial w_{1}}$ \\
& $\lambda\left(w_{0 x}\right)=\lambda_{0}\left(w_{0 x}+q\right)^{\frac{1+s}{p}-1}$ & \\
\hline
\end{tabular}




\section{A physical application}

Let us consider a homogeneous viscoelastic bar of uniform cross-section and assume the material to be a nonlinear Kelvin solid. The classical equation of motion (the constant density is normalized to 1 and the mass forces are neglected)

$$
w_{t t}=\tau_{x}
$$

assuming a stress-strain relation of the following form:

$$
\tau=\sigma\left(w_{x}\right)+\lambda\left(w_{x}\right) w_{x t},
$$

where $\tau$ is the stress, $x$ the position of a cross-section in the homogeneous rest configuration of the bar, $w(t, x)$ the displacement at time $t$ of the section from its rest position, $\sigma\left(w_{x}\right)$ the elastic tension ( $w_{x}$ is the strain), and $\lambda\left(w_{x}\right) w_{x t}$ is the viscosity component of the stress, reduces to (2).

Let us consider the following form of the tension $\sigma\left(w_{x}\right)$ :

$$
\sigma\left(w_{x}\right)=\sigma_{0} \log \left(1+w_{x}\right)
$$

which was suggested by Capriz $[15,16]$.

So, we fall in Case II of Table 1 with the following identifications:

$$
f_{0}=\sigma_{0}, \quad p=-2, \quad q=1 \text {. }
$$

In this case, the approximate Lie operator $X_{8}$ assumes the form

$$
X_{8}=x \frac{\partial}{\partial x}-\left(w_{0}+2 x\right) \frac{\partial}{\partial w_{0}}+s w_{1} \frac{\partial}{\partial w_{1}}
$$

and from the corresponding invariant surface conditions, we obtain the following representation for the different terms in the expansion of $w$ :

$$
\begin{aligned}
& w_{0}=\frac{\psi(t)}{x}-x, \\
& w_{1}=\chi(t) x^{s},
\end{aligned}
$$

which give the form of an invariant solution approximate at the first order in $\varepsilon$.

The functions $\psi$ and $\chi$ must satisfy the following system of ODEs to which, after (44), the system (9)-(10) is reduced through (47) and (48) (we have chosen $s=-5$ ):

$$
\begin{aligned}
& \psi_{t t}+2 \sigma_{0}=0, \\
& \chi_{t t}+20 \sigma_{0} \frac{\chi}{\psi}+4 \lambda_{0} \psi \psi_{t}=0 .
\end{aligned}
$$

After solving (49)-(50) and taking (47)-(48) into account, we have

$$
w_{0}=-\sigma_{0} \frac{t^{2}}{x}-x
$$




$$
w_{1}=-\frac{8}{81} \sigma_{0}^{2} \lambda_{0} t^{5}(9 \log t-1)
$$

Therefore, the invariant solution up to the first order in $\varepsilon$ is

$$
w(t, x, \varepsilon)=-\sigma_{0} \frac{t^{2}}{x}-x-\varepsilon \frac{8 \sigma_{0}^{2} \lambda_{0} t^{5}(9 \log t-1)}{81 x^{5}}+\mathcal{O}\left(\varepsilon^{2}\right) .
$$

We have an unperturbed state represented by a stretching modified by the viscosity effect.

\section{Conclusions}

In this paper we perform the group analysis of the nonlinear wave equation with a small dissipation (2) in the framework of the approximate method proposed in [12,13]. In order to remove the 'drawback' of the method proposed by Fushchich and Shtelen [11], we introduce, according to the perturbation theory, the expansions of the dependent variables in the one-parameter Lie group of infinitesimal transformations of equation (2). Equating to zero the coefficients of zero and first degree powers of $\varepsilon$, we obtain an approximate Lie operator which permits to solve in hierarchy the invariance condition of the system (9)-(10) starting from the classification of unperturbed nonlinear wave equation (3). The proposed strategy is consistent with the perturbation point of view and can be generalized in a simple way to the higher orders of approximation in $\varepsilon$.

Competing interests

The authors declare that they have no competing interests.

Authors' contributions

The authors wrote this paper in collaboration and with the same responsibility. All authors read and approved the final version of the manuscript.

\section{Author details}

'Facoltà di Ingegneria e Architettura, Università di Enna 'Kore', Cittadella Universitaria, Enna, 94100, Italy. ${ }^{2}$ Dipartimento di Matematica e Informatica, Università di Catania, Viale Andrea Doria 6, Catania, 95125, Italy.

\section{Acknowledgements}

MR acknowledges the support of GNFM through the project 2012 Metodologie di tipo analitico e numerico per lo studio di problemi iperbolici ed iperbolico-parabolici di natura ondosa.

Received: 9 March 2013 Accepted: 20 May 2013 Published: 10 June 2013

\section{References}

1. Dafermos, CM: The mixed initial-boundary value problem for the equations of non-linear viscoelasticity. J. Differ. Equ. 6, 71 (1969)

2. MacCamy, RC: Existence, uniqueness and stability of $u_{t t}=\frac{\partial}{\partial x}\left[\left(u_{x}\right)+\left(u_{x}\right) u_{x t}\right]$. Indiana Univ. Math. J. 20, $231(1970)$

3. Rajagopal, KR, Saccomandi, G: Shear waves in a class of nonlinear viscoelastic solids. Q. J. Mech. Appl. Math. 56, 311 (2003)

4. Ruggieri, M, Valenti, A: Symmetries and reduction techniques for dissipative models. J. Math. Phys. 50, 063506 (2009)

5. Ruggieri, M, Valenti, A: Exact solutions for a nonlinear model of dissipative media. J. Math. Phys. 52, 043520 (2011)

6. Ruggieri, M: Kink solutions for class of generalized dissipative equations. Abstr. Appl. Anal. 2012, 237135 (2012). doi:10.1155/2012/237135

7. Ruggieri, M, Valenti, A: Group analysis of a nonlinear model describing dissipative media. In: Ibragimov, NH, Sophocleous, C, Damianou, PA (eds.) Proceedings of MOGRAN X, p. 175 (2005)

8. Ruggieri, M, Valenti, A: Symmetries and reduction techniques for a dissipative model. In: Monaco, R, Mulone, G, Rionero, S, Ruggeri, T (eds.) Proceedings of WASCOM 2005, p. 481. World Scientific, Singapore (2006)

9. Ruggieri, M, Valenti, A: Symmetry analysis of viscoelastic model. In: Manganaro, N, Monaco, R, Rionero, S (eds.) Proceedings of WASCOM 2007, p. 514. World Scientific, Singapore (2008)

10. Baikov, VA, Gazizov, RK, Ibragimov, NH: Approximate symmetries of equations with a small parameter. Mat. Sb. 136 435 (1988) (English transl. in: Math. USSR Sb. 64, 427-441 (1989))

11. Fushchich, WI, Shtelen, WM: On approximate symmetry and approximate solutions of the non-linear wave equation with a small parameter. J. Phys. A, Math. Gen. 22, L887-L890 (1989)

12. Valenti, A: Approximate symmetries for a model describing dissipative media. In: Ibragimov, NH, Sophocleous, $C$, Damianou, PA (eds.) Proceedings of MOGRAN X, p. 236 (2005) 
13. Valenti, A: Approximate symmetries of a viscoelastic model. In: Manganaro, N, Monaco, R, Rionero, S (eds.) Proceedings of WASCOM 2007, p. 582. World Scientific, Singapore (2008)

14. Ibragimov, NH: CRC Handbook of Lie Group Analysis of Differential Equations. CRC Press, Boca Raton (1994)

15. Capriz, G: Waves in strings with non-local response. In: Mathematical Problems in Continuum Mechanics, Trento (Italy), 12-17 January 1981. Internal Report No. 13, CIRM (1981)

16. Capriz, G: Non-linear dynamics of a taut elastic string. In: Anile, AM, Cattaneo, G, Patano, P (eds.) Atti delle Giornate di Lavoro su Onde e Stabilità nei Mezzi Continui, Cosenza (Italy), 6-11 June 1983. Quaderni del Consiglio Nazionale delle Ricerche (CNR): Gruppo Nazionale per la Fisica Matematica (GNFM). Tipografia dell'Università, Catania (1986)

doi:10.1186/1687-2770-2013-143

Cite this article as: Ruggieri and Valenti: Approximate symmetries in nonlinear viscoelastic media. Boundary Value Problems 2013 2013:143.

Submit your manuscript to a SpringerOpen ${ }^{\circ}$ journal and benefit from:

- Convenient online submission

- Rigorous peer review

- Immediate publication on acceptance

- Open access: articles freely available online

- High visibility within the field

- Retaining the copyright to your article

Submit your next manuscript at $\gg$ springeropen.com 